\title{
Ultrasound-Guided Percutaneous Scaphoid Pinning: Operator Variability and Comparison with Traditional Fluoroscopic Procedure
}

\author{
Maarten Beek ${ }^{1}$, Purang Abolmaesumi ${ }^{2,3}$, Suriya Luenam ${ }^{4}$, \\ Richard W. Sellens ${ }^{1}$, and David R. Pichora ${ }^{1,4}$ \\ ${ }^{1}$ Department of Mechanical and Materials Engineering, Queen's University, Canada \\ ${ }^{2}$ School of Computing, Queen's University, Canada \\ ${ }^{3}$ Department of Electrical and Computer Engineering, Queen's University, Canada \\ ${ }^{4}$ Division of Orthopaedic Surgery, Kingston General Hospital, Canada \\ purang@cs . queensu.ca
}

\begin{abstract}
This paper reports on pilot laboratory experiments with a recently proposed surgical procedure for percutaneous screw insertion into fractured scaphoid bones using ultrasound guidance. The experiments were intended to determine the operator variability of the procedure and its performance in comparison with a traditional pinning procedure using fluoroscopy. In the proposed procedure, a three-dimensional surface model is created from pre-operative computed tomography images and intra-operatively registered to the patient using ultrasound images. A graphical interface that communicates with an optical camera tracking the surgical tools, guides the surgeon during the procedure in real time. The results of the experiments revealed non-significant differences between operators for the error in the entry location of the drill hole $(\mathrm{p}=0.90)$; however, significant differences for the exit location $(\mathrm{p}<0.05)$. Comparison with the traditional pinning procedure shows that the outcome of the recently proposed procedure appears to be more consistent.
\end{abstract}

\section{Introduction}

The scaphoid bone (Figure 1) is the most frequently fractured bone in the wrist, representing about $90 \%$ of all wrist fractures 1 . In general, the bone is fractured through its relatively narrow waist [2]. It is generally accepted that immobilization in a short-arm cast needs to last for ten to twelve weeks 113. When the proximal pole is involved, this immobilization period is often prolonged. Due to the poor blood supply of the proximal part of the bone, complications like malor non-unions are common; Mink van der Molen and co-workers reported that $12.8 \%$ of all scaphoid fracture cases in their study, of which $98 \%$ were initially treated by cast immobilization, eventually underwent operative treatment [1]. The outcomes of internal fixation of the scaphoid show high patient satisfaction and good wrist mobility [2]. In case of complications, pinning of the scaphoid is often the only remaining treatment option. An open approach for scaphoid pinning facilitates an accurate placement of the screw, because the bone and 


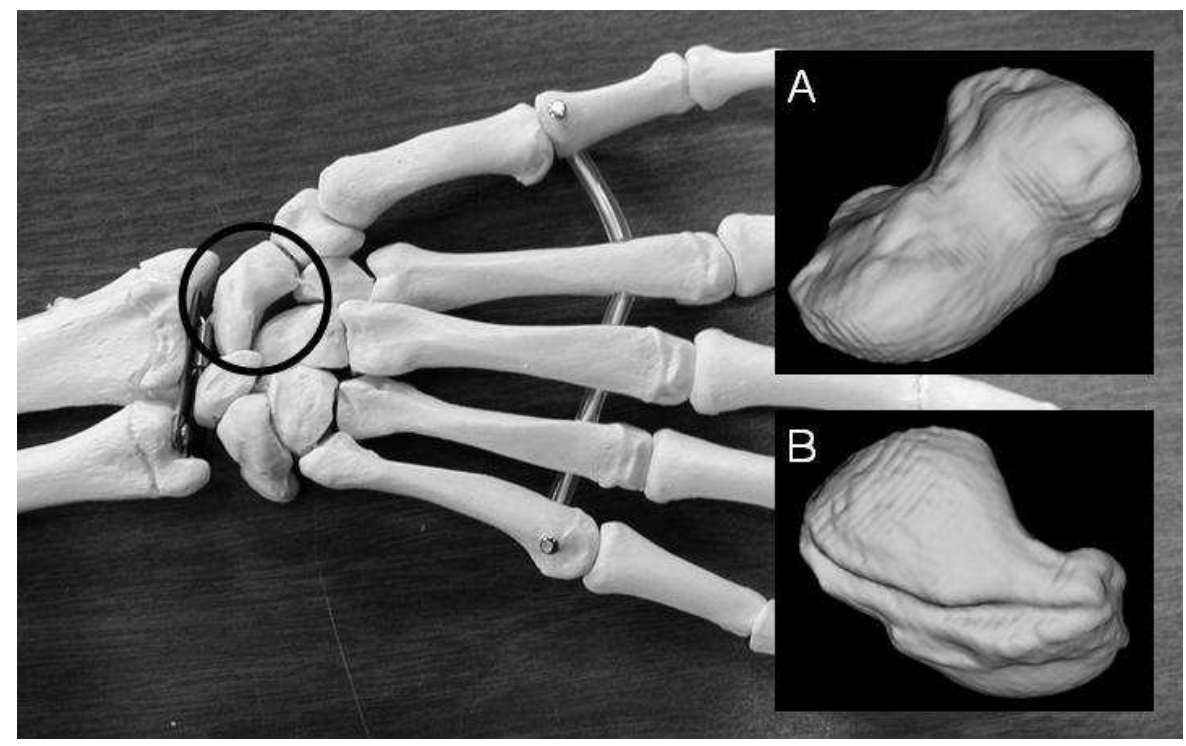

Fig. 1. Dorsal view of a plastic model of the bones of a human hand showing the anatomical position of the scaphoid bone (circle). Both inserts show the threedimensional surface model of a typical scaphoid. A: palmar view; B: dorsal view.

the surrounding soft tissues are directly visible to the surgeon. However, open surgery inevitably involves some degree of soft tissue damage and an increased risk for infections. The open approach also violates several key ligaments, which may result in scaphoid instability [4. Performing the procedure percutaneously reduces many of the drawbacks associated with an open surgery. However, insertion of the screw is challenging, due to the small size of the bone and the lack of direct vision of the surgical tools with respect to the targeted anatomy. The traditional percutaneous pinning procedure generally requires many intra-operative fluoroscopic images to ensure an accurate screw placement. For operating room staff and patients this means additional exposure to harmful ionizing radiation. Another drawback is the two-dimensional nature of fluoroscopic images, which limits the three-dimensional positioning of surgical tools. Furthermore, the close proximity of other carpal bones in the wrist joint interferes with the acquisition of clear lateral fluoroscopic scaphoid images. Recently, studies on arthoscopically assisted internal fixation of the scaphoid have been published [56]. The outcomes of this approach are good and the procedure even enables the detection of soft tissue lesions. However, fluoroscopy was nonetheless applied to verify the position and orientation of the inserted K-wires in both studies.

In an earlier publication, we proposed a new procedure for percutaneous scaphoid pinning which involves pre-surgery planning using computed tomography (CT) images and intra-operative guidance using three-dimensional ultrasound (US) 7]. A graphical interface displays the surface model with the surgical plan, and the surgical tools in real time to guide the surgeon during screw 
insertion. This paper reports on the results of laboratory experiments into the operator variability of the newly proposed procedure and its performance in comparison with the traditional percutaneous procedure using fluoroscopy.

\section{Materials and Methods}

Two human scaphoid phantoms (No. 1009, Sawbones Inc., Vashon WA) were attached to $5 \times 10 \mathrm{~cm}$ Plexiglas sheets after flattening their dorsal side. Three alumina beads (diameter: approximately $3 \mathrm{~mm}$ ) were mounted to each phantom bone to verify the accuracy of each procedure. The goal in the experiments was to drill a hole through each phantom bone according to a consistent surgical plan. This plan was chosen through the geometric center of the surface model in the direction of the model's main axis, determined from a principal component analysis. To determine the operator variability of the proposed procedure, two different operators were asked to drill into eight different phantoms. Furthermore, one of the operators was also asked to drill into an additional eight phantoms in the traditional way using fluoroscopy to enable comparison of the proposed procedure with the traditional fluoroscopic procedure. Statistical analyses to determine significant differences between operators and performances were performed using independent two-tailed t-tests. Equality of variances was tested using the Levene's test.

\subsection{Generation of Surface Models}

Each Plexiglas sheet with the scaphoid phantoms and beads was scanned using a LightSpeed Plus CT scanner (GE Medical Systems, Waukesha WI) at Kingston General Hospital. The in-plane resolution of the images was $0.19 \times 0.19 \mathrm{~mm}$ and the images were $1.25 \mathrm{~mm}$ apart (interpolated to $0.625 \mathrm{~mm}$ ). Software developed at Queen's University was used to segment the geometry of the bone phantoms from the CT images and to create three-dimensional surface models of the phantoms using a marching cubes algorithm. The locations in CT coordinates of the alumina beads were determined from the CT images and recorded.

\subsection{Traditional Procedure Using Fluoroscopy}

Each scaphoid phantom was covered by a piece of opaque foam to simulate the percutaneous character of the procedure. The amount of fluoroscopic images needed to achieve an accurate drill hole according to the surgical plan, was recorded for each phantom. After the experiments each Plexiglas sheet was mounted to a stainless steel jig equipped with an optical target serving as frame of reference. Using a Certus optical camera (Northern Digital Inc., Waterloo ON) and a calibrated stylus probe, the position of the alumina beads and of the entry and exit of each realized drill hole was measured after the experiment. Using the alumina beads, the surface model of each phantom was registered to the frame of reference, enabling the determination of the error between the planned and realized drill holes. 


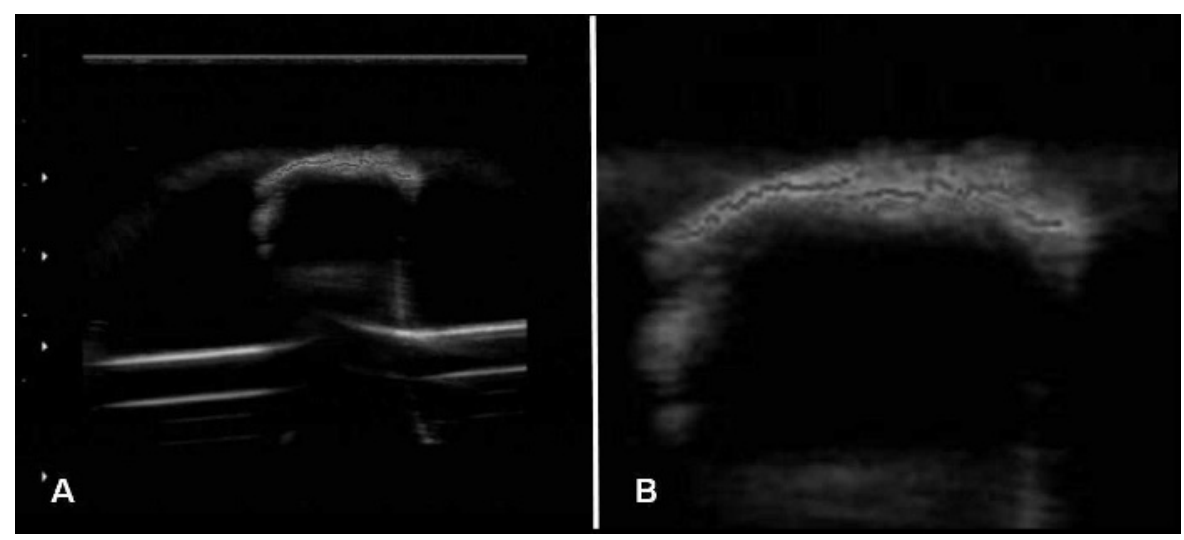

Fig. 2. Semi-automatic segmentation of ultrasound images. A: typical segmentation result; B: close-up of A. The darker points in the bright band represent the segmentation.

\subsection{Proposed Procedure Using Ultrasound}

In this part of the study, the same Certus optical camera was used to track the optical targets in real time. Each Plexiglas sheet with the phantom bones was rigidly mounted to the stainless steel jig with the reference optical target. A $12 \mathrm{MHz}$, one-dimensional array US transducer (GE Medical Systems, Waukesha WI) and a surgical drill were also equipped with optical targets. Due to the amount of time involved, the US transducer was pre-calibrated using an $\mathrm{N}$-wire phantom and the error of this calibration method proved to be $0.64 \mathrm{~mm}[8$. The US transducer was handled carefully throughout the experiments to avoid jeopardizing its calibration. For each experiment, the drill was re-calibrated: The tip position of the drill bit was determined by pivoting 9 and the direction of the drill bit was determined from this tip position and the center of the drill chuck which was measured with a calibrated stylus probe before inserting the drill bit 7. The tip position accuracy proved to be better than $0.3 \mathrm{~mm}$ after calibrating by means of pivoting [7]. US images were used to register the surface models to the frame of reference. After the jig was placed in a tub of room-temperature water, US images were captured through a frame-grabber, which was synchronized with the optical camera. The US images were semi-automatically segmented (Figure 2): A few (normally less than five) seed pixels are selected in each image by the operator using a mouse pointer. Subsequently, after linear interpolation of the seeds, the pixels are moved up and down within a small vertical band and the ones with the highest intensity are selected. These pixels are finally converted into a point cloud (using the US calibration data and the position and orientation data from the optical camera). The surface model was then registered to the points segmented from the US images using an iterative closest point (ICP) algorithm [10. The mean of the surface registration error was $0.53 \mathrm{~mm}$ and the mean of the fiducial registration error was $2.7 \mathrm{~mm} \mathrm{[7].} \mathrm{Two} \mathrm{operators} \mathrm{were} \mathrm{asked}$ to drill one hole through scaphoid phantoms according to the surgical plan while 


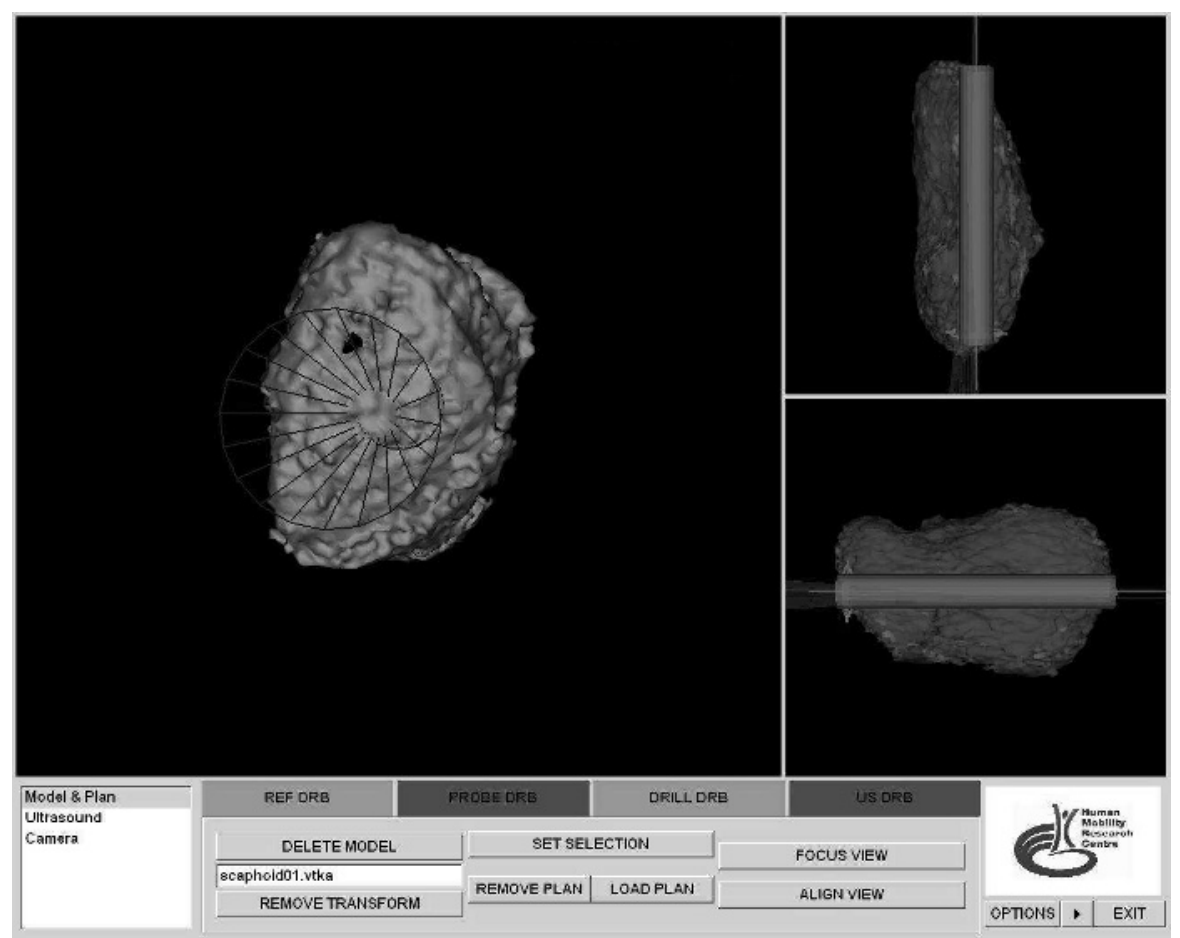

Fig. 3. Graphical interface to guide the surgeon during percutaneous scaphoid pinning. The interface displays three orthogonal views of which the larger one can be aligned with the direction of the surgical plan. The surgical plan is represented by the cylinder and the surgical drill by the wireframe cone.

looking at a computer monitor. In the monitor, the graphical interface was displayed, showing the surface model and graphical representations of the surgical plan and surgical drill in real-time (Figure 3). The position of the entry and exit of each realised drill hole was measured using a calibrated stylus probe.

\section{Results}

The mean error of the entry location between the planned and realized drill hole was $1.89 \mathrm{~mm}$ and $1.93 \mathrm{~mm}$ for operator $1(n=13)$ and operator $2(n=8)$, respectively. Statistical analysis showed that this difference was not significant $(\mathrm{p}=0.90)$. However, for the mean error of the exit location these numbers were $3.04 \mathrm{~mm}$ and $1.99 \mathrm{~mm}$, respectively. This difference appeared to be significant $(\mathrm{p}<0.05)$.

For the traditional pinning procedure, on average, 7.6 fluoroscopic images were needed for each phantom (range: 5-11). For a comparison of the proposed with the traditional procedure, the reader is referred to Figure 4. The mean of the distance between the realized and planned entry of the drill hole was $2.63 \mathrm{~mm}$ 


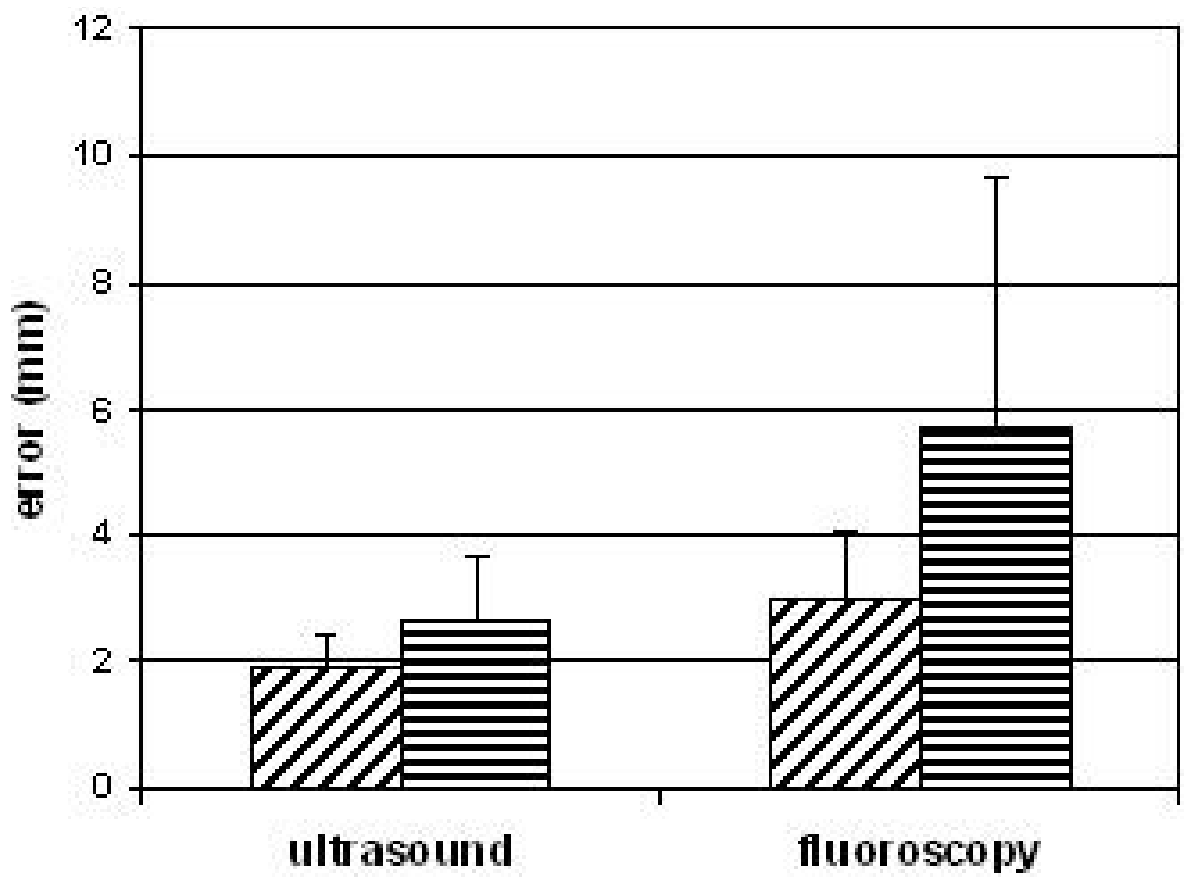

Fig. 4. Comparison of the mean of the errors (error bars represent one standard deviation) in the entry location (diagonal lines) and exit location (horizontal lines) of the realized drill hole for the proposed scaphoid pinning procedure using ultrasound and the traditional approach using fluoroscopy $(n=8)$.

(standard deviation: $1.01 \mathrm{~mm}, \mathrm{n}=8$ ). This value was $5.73 \mathrm{~mm}$ for the exit of the drill hole (standard deviation: $3.95 \mathrm{~mm}, \mathrm{n}=8$ ). For the newly proposed procedure, the same operator realized values of $1.89 \mathrm{~mm}$ (standard deviation: $0.55, \mathrm{n}=8$ ) and $3.04 \mathrm{~mm}$ (standard deviation: $1.05, \mathrm{n}=8$ ) for the means of the errors in the entry and exit of the realized drill hole, respectively. The differences did not appear to be significant. The Levene's test for equality variance indicated a difference that approached significance for the error of the entry of the drill hole.

\section{Conclusions}

A new percutaneous scaphoid pinning procedure was proposed, that uses a threedimensional surface model of the bone from pre-operative CT images and intraoperative ultrasound guidance. Since the scaphoid bone articulates with five other bones, lots of its surface is covered with articulating cartilage. In order to prevent cartilage damage that might introduce osteoarthritis, a distance of maximally $2 \mathrm{~mm}$ from the bone's main axis is acceptable for screw insertion. The results of this study show that this tight accuracy requirement can be met. Advantages of the proposed procedure are a reduced risk of infections and minimal 
soft tissue damage due to its percutaneous nature. A recent study showed that the radiation exposure to the hands of the surgeon in every fluoroscopic procedure is on average comparable with a chest x-ray (approximately $20 \mathrm{mrem}$ ), limiting the recommended number of cases to about 2,500 per year [1]. To the patient the effective radiation involved with the pre-operative CT scanning will be negligible since the volume which is scanned is small and no major organs are within this volume. Due to the complex anatomy of the wrist joint, the accompanying ability to plan the surgery in advance can also be considered as a plus for the proposed procedure.

The operator variability of the proposed procedure appeared to be minimal. The significant difference between operators in the error of the exit location of the realized drill hole might be due to differences in operator experience with the guidance interface. The small sample size of this pilot study may be the main reason for the lack of differences that are statistically significant when comparing the newly proposed ultrasound-guided procedure with the traditional fluoroscopic approach. However, the fact that the difference between the variance for the entry of the drill hole approaches significance might be indicative for the improved consistency of the ultrasound-guided procedure. Segmentation and registration were not real time in this study. However, it appeared that between 15 and 20 US images were required for obtaining a sufficiently accurate registration. The total amount of time involved between capturing the first US image and finishing the registration was nonetheless acceptable (less than 3 minutes). It is well-known that registration algorithms, like the iterative closest point method [10], need a sufficiently accurate initial estimation to avoid getting stuck in a wrong local minimum. Presently, this initial estimation is obtained by manual manipulation of the surface model with respect to the points segmented from the US images. A future version of the graphical interface might incorporate the selection of pre-determined landmarks on the patient using a calibrated stylus probe to facilitate a landmark registration as a first estimate. Another option is to try all orientations that can be achieved by flipping the model about its main axes (just eight) and assuming that the registration with the smallest average distance between the points and the model is the correct registration.

The experiments described in this paper are pilot experiments enabling more realistic experiments in the near future. During an actual surgery, the acquisition of clear lateral fluoroscopic images of the scaphoid is virtually impossible due to the close proximity of the other carpal bones. Experiments using fluoroscopic guidance on isolated scaphoids might, therefore, result in incorrect conclusions. Because the reference optical target cannot be mounted directly to the scaphoid in an actual pinning procedure, the bone has to be registered with respect to an optical target close to the surgical target area. Since the ligaments in the wrist joint are tight and the hand is generally positioned in a pose that locks the scaphoid during screw insertion, we expect the mobility of the scaphoid to be negligible, but this has yet to be verified. 


\section{Acknowledgment}

We thank the staff at Imaging Services at Kingston General Hospital for their assistance in obtaining CT images. We also thank Heather Grant for assisting in the statistical analyses.

\section{References}

1. Mink van der Molen, A., Groothoff, J., Visser, G., Robinson, P., Eisma, W.: Time off work due to scaphoid fractures and other carpal injuries in the netherlands in the period 1990 to 1993. Journal of Hand Surgery 24B (1999) 193-198

2. Daeke, W., Wieloch, P., Vergetis, P., Jung, M., Martini, A.: Occurrence of carpal osteoarthritis after treatment of scaphoid nonunion with bone graft and herbert screw: a long-term follow-up study. Journal of Hand Surgery 30A (2005) 923-931

3. Saeden, B., Tornkvist, H., Ponzer, S., Hoglund, M.: Fracture of the carpal scaphoid: A prospective, randomised 12-year follow-up comparing operative and conservative treatment. Journal of Bone and Joint Surgery 83B (2001) 230-234

4. Filan, S., Herbert, T.: Herbert screw fixation of scaphoid fractures. Journal of Bone and Joint Surgery 78B (1996) 519-529

5. Shih, J., Lee, H., Hou, Y., Tan, C.: Results of arthroscopic reduction and percutaneous fixation for acute displaced scaphoid fractures. Arthroscopy 21 (2005) $620-626$

6. Slade, J., Gutow, A., Geissler, W.: Percutaneous internal fixation of scaphoid fractures via an arthroscopically assisted dorsal approach. Journal of Bone and Joint Surgery 84A (2002) 21-36

7. Beek, M., Abolmaesumi, P., Chen, T., Sellens, R., Pichora, D.: Percutaneous scaphoid pinning using ultrasound guidance. In: Cleary, K. R., Galloway, R. L. Jr. (Eds.) Medical Imaging 2006: Visualization, Image-Guided Procedures, and Display. (2006)

8. Chen, T., Abolmaesumi, P., Pichora, D., Ellis, R.: A system for ultrasound-guided computer-assisted orthopaedic surgery. Computer Aided Surgery 10 (2005) 281292

9. Schmerber, S., Chassat, F.: Accuracy evaluation of a cas system: Laboratory protocol and results with $6 \mathrm{~d}$ localizers, and clinical experiments in otorhinolaryngology. Computer Aided Surgery 6 (2001) 1-13

10. Besl, P., McKay, N.: A method for registration of 3-d shapes. IEEE Transactions on Pattern and Machine Intelligence 14 (1992) 239-256

11. Singer, G.: Radiation exposure to the hands from mini c-arm fluoroscopy. Journal of Hand Surgery 30A (2005) 795-797 